\title{
Assessing the Role of Potential Biomarkers in Antimony Susceptible and Resistant Clinical Isolates of L. donovani from India \\ Mahendra Maharjan ${ }^{1,2 *}$, Swati Mandal' ${ }^{1}$, Rentala Madhubala ${ }^{1}$ \\ ${ }^{1}$ School of Life Sciences, Jawaharlal Nehru University, New Delhi, 110067, India. \\ 2 Central Department of Zoology, Tribhuvan University, Kathmandu, Nepal.
}

\begin{abstract}
Failure of antimonial drugs, the mainstay therapy for leishmaniasis has become an escalating problem in the treatment of Indian leishmaniasis. Using 14 clinical isolates from both visceral (VL) and post-kala-azar dermal leismaniasis (PKDL) patients, we have examined the role of ATP-binding cassette transporter (ABC transporter) gene, multidrug resistant protein A (MRPA) and two building blocks of the major thiol, trypanothione namely, ornithine decarboxylase gene (ODC) (a rate limiting enzyme in the polyamine biosynthesis) and $\gamma^{-}$ glutamylcysteine synthetase ( $\gamma$-GCS) (a rate limiting enzyme in glutathione biosynthesis) in antimony resistance. Amplification of these three genes was observed in some but not all clinical isolates. Increased expression of the three RNAs as determined by real-time PCR was observed in all SAG-R clinical isolates. Significant increase in cysteine and glutathione levels was observed in the resistant isolates. Our studies report the underlying mechanism of antimony resistance in the clinical isolates.
\end{abstract}

Key words: $A B C$ transporter, ornithine decarboxylase, $\gamma$ - glutamylcysteine synthetase, antimony resistance.

*Corresponding Author

Email: maharjan.m@gmail.com

\section{Introduction}

The protozoan parasite Leishmania is the causative agent of kala-azar and is responsible for a variety of clinical manifestations. It causes a wide spectrum of diseases ranging from the simple self healing cutaneous form to the debilitating visceral form. Visceral leishmaniasis (VL) is caused by L. donovani in the Indian subcontinent. Pentavalent antimonials $(\mathrm{SbV})$ are the first line of drug used in the treatment against all forms of leishmanial infections [1,2]. Resistance to this drug is becoming a major barrier in the treatment of VL in many endemic regions particularly in India [3]. Kala-azar transmission in India is thought to be anthroponotic and postkala-azar dermal leismaniasis (PKDL) patients are considered to serve as a source for new outbreaks [4]. The post-kala-azar dermal leismaniasis (PKDL) is a sequel to VL in India

and Sudan; the disease develops months to years after the patient's recovery from VL [5].

The mechanism of action of sodium antimony gluconate (SAG) remained an enigma for more than 60 years of its effective use against all forms of leishmaniasis. It is generally agreed that pentavalent form $(\mathrm{SbV})$ is reduced to a more toxic trivalent form (SbIII) which constitutes the active form of the drug against the parasite [6]. Molecules possibly implicated in reduction of $\mathrm{SbV}$ to SbIII include host and parasite thiols and two newly discovered parasite enzymes thioldependent reductase (TDR1) and arsenate reductase (ACR2) [7-9]. A loss of drug activation is reported to lead to resistance [10]. The route of entry of $\mathrm{SbV}$ into Leishmania cells is still unknown but SbIII has been reported to be transported in Leishmania through aquaglyceroporin (AQP1) [11,12]. Recent evidence has suggested that part of the mode of 
action of $\mathrm{SbV}$ could be in depleting the cells of its reduced thiols [13]. Trypanothione (TSH), a major reduced thiol of Leishmania is a $\mathrm{N}^{1}, \mathrm{~N} 8$ bisglutathione spermidine conjugate. It is thought to bind to the active reduced form of the metal. These metal-trypanothione conjugates are either sequestered into an intracellular organelle by the $A B C$ transporter MRPA or extruded outside the cell by an efflux pump [13-16]. A number of candidate genes associated with increased thiol concentration have been described in Leishmania laboratory mutants. Resistance was induced in these mutants in vitro in the presence of antimony related metals such as arsenic or antimony $[2,17,18]$.

However, till date it remains unclear as to whether similar mechanisms can be extrapolated to clinical isolates from geographical zones with a high incidence of primary antimony resistance. To address this question, we have characterized both the VL and the PKDL isolates from India and report that diverse mechanisms of resistance are operative in these isolates. This work aims at characterizing possible biomarkers for monitoring antimonial resistant visceral leishmaniasis and post-kala-azar dermal leismaniasis in the field isolates.

In the present study, we report the role of thiols and also assessed the role of $\mathrm{ABC}$ transporter (MRPA), ornithine decarboxylase (ODC) and $\gamma$ glutamylcysteine synthetase ( $\gamma$-GCS) genes as potential biomarkers for monitoring antimonial resistance in Indian leishmaniasis.

\section{Materials and Methods}

\section{Parasite and culture conditions}

Promastigotes of Leishmania donovani clones, AG83 (MHOM/IN/80/AG83), 2001, MC4, MC7, MC8 and MC9 were isolated from patients with VL and strains, RK1, MS2, NR3A, RMP8 (HM/IN/RMP-8), RMP-19 (HM/IN/RMP-19), RMP142 (HM/IN/RMP-142), RMP155 (HM/IN/RMP-155) and RMP240 (HM/IN/RMP-240) used in the present study were isolated from patients with PKDL [19]. Clinical isolates obtained from VL and PKDL patients who responded to SAG chemotherapy were designated as SAG-S (SAG-sensitive) whereas isolates from VL and PKDL patients who did not respond to SAG were designated as SAG-R (SAG-resistant). SAG-sensitive strains, AG83-S, 2001-S, MC7-S, RK1-S, MS2-S and the nine SAG-R isolates, MC4-R MC8-R, MC9-R, NR3A-R, RMP8-R, RMP19-R, RMP142-R, RMP155-R and RMP240-R have been characterized earlier [20]. Clinical history of the patient infected with the strain RK1-S showed that the interval between the cure of VL and the onset of PKDL was 2.5 years where as in the case of PKDL patients infected with the strains, MS2$S$ and NR3A-R, the interval between the cure of VL and the onset of PKDL was 7 and 11 years respectively. The interval between the cure of VL and the onset of PKDL for the remaining isolates is not known. The clinical isolates were maintained in vitro in the absence of the drug pressure. Promastigotes were routinely cultured at $22^{\circ} \mathrm{C}$ in modified M-199 medium (Sigma, USA) supplemented with $10 \%$ heat-inactivated fetal bovine serum (FBS; Gibco/BRL, Life Technologies Scotland, UK) and $0.13 \mathrm{mg} / \mathrm{mL}$ penicillin and streptomycin. This study has the approval of the Institutional level ethics committee.

\section{DNA and RNA manipulations}

Chromosomes of the clinical isolates were separated by pulse field gel electrophoresis (PFGE) in which low melting agarose blocks, containing embedded cells $\left(10^{8} / \mathrm{ml}\right.$ log phase promastigotes) were electrophoresed in a contour clamped homogeneous electric field apparatus (CHEF DRIII, Bio-Rad) as reported earlier [20]. Mid log phase promastigotes $(\sim 2 \mathrm{x}$ $10^{9}$ cells) of all the field isolates were used for isolation of genomic DNA. $5 \mu \mathrm{g}$ of genomic DNA was digested with HindIII enzyme and subjected to electrophoresis. Total RNA was isolated from promastigotes $\left(2 \times 10^{8}\right.$ cells) using RNAeasy Plus Mini Kit (Qiagen). Standard protocols were followed for Southern hybridization [21]. DNA probes used in the present study included a 400-bp MRPA fragment (released from plasmid PM12 that was 
digested with BamHI and PstI), a 2.3-kb үGCS fragment - (derived from plasmid pspahygroayGCS digested with HindIII and XbaI), a $2.0-\mathrm{kb}$ $O D C$-full length probe (derived from plasmid pspahygroa-ODC digested with HindIII and $\mathrm{XbaI}$ ) and a 1.6-kb 5'-PTR1 probe derived from plasmid (psp72-Y-hygro-5'-PTR1).

\section{cDNA synthesis and real time RT- PCR}

Total RNA was isolated from $10^{8}$ Leishmania cells in the mid-log phase of growth using the RNeasy Plus Mini Kit (Qiagen) as described by the manufacturer. Quality and quantity of the RNA were determined using the RNA 6000 Nano Lab chip kit on the Bio-analyzer 2100 (Agilent Technologies). The sequences of the primers for MRPA are forward 5' GCGCAGCCGTTTGTGCTTGTGG-3' and reverse 5'-TTGCCGTACGTCGCGATGGTGC-3' and for the glyceraldehyde 3-phosphate dehydrogenase (GAPDH) control forward 5'GAAGTACACGGTGGAGGCTG and reverse $5^{\prime}$ CGCTGATCACGACCTTCTTC primers. The sequences of the primers for $O D C$ are forward 5'- GATGGTGCGCCCTTACTTTGC-3'and reverse 5'-TTCCATCTCCAGCGGGTTGTCC-3', and for the $r G C S$, forward $5^{\prime}$ CATTGGCTGGCGCGTTGAGTTC-3' and reverse 5'-ATGTGCGCGGCCCATATTCTCG -3' primer. Complementary DNAs from promastigotes were synthesized from $500 \mathrm{ng}$ of total RNA using the AccuSuperscript High Fidelity RT-PCR kit (Stratagene, La Jolla) and Oligo (dT) 18 primers following manufacturer's instructions. Real-time PCR was performed in triplicate in $25 \mu \mathrm{l}$ volumes using QuantiFast SYBR Green PCR Master Mix (Qiagen) in an Applied Biosystem 7500. Reactions were run using the following thermal profile: initial denaturation at $95^{\circ} \mathrm{C}$ for $5 \mathrm{~min}$ followed by 40 cycles with denaturation at $95^{\circ} \mathrm{C}$ for $30 \mathrm{~s}$, annealing at $62^{\circ} \mathrm{C}$ for $1 \mathrm{~min}$ and extension at $72^{\circ} \mathrm{C}$ for $20 \mathrm{~s}$. The PCR was followed by a melt curve analysis to ascertain that the expected products were amplified. The relative amount of PCR products generated from each primer set was determined based on the threshold cycle
(Ct) value and amplification efficiencies and was normalized by dividing the values by the relative amount of the GAPDH gene used as a control.

\section{Transfection and overexpression of the MRPA} and $\boldsymbol{\gamma}$-GCS gene

Episomal Leishmania expression vectors, pGL$\alpha N E O \alpha L U C$ containing luciferase encoding DNA and neomycin phosphotransferase selectable marker, pspahygro $\alpha-\gamma$ GCS containing coding sequence for heavy subunit of $\gamma$ GCS with hygromycin phosphotransferase as selectable marker and pGEM72f- $\alpha$ neo $\alpha$-MRPA containing MRPA coding DNA and neomycin phosphotransferase as selectable marker were obtained as gifts from Prof. Marc Ouellette, Centre de Recherche en Infectiologie du Centre de Recherche du CHUL, Universite Laval, Quebec, Canada. Twenty $\mu \mathrm{g}$ of each construct was transfected into L. donovani promastigotes by electroporation. Electroporation was done with a single pulse with the following parameters $450 \quad \mathrm{~V}, \quad 500 \mu \mathrm{F} \quad$ (Bio-Rad). Transfectants were selected for resistance to either G418 $(40 \mu \mathrm{g} / \mathrm{ml})$ or hygromycin B $(80$ $\mu \mathrm{g} / \mathrm{ml}$ ) as described earlier [22].

Chemosensitivity profiles of SAG-S and SAG-R strains in an amastigote macrophage model

Stationary phase Leishmania promastigotes expressing the luciferase gene (pGL$\alpha$ NEO $\alpha$ LUC) and pspahygro $\alpha-\gamma$ GCS containing coding sequence for heavy subunit of $\gamma$ GCS with hygromycin phosphotransferase as selectable marker or pGEM72f- $\alpha$ neo $\alpha-M R P A$ containing MRPA coding DNA and neomycin phosphotransferase as selectable marker were infected into J774A.1 macrophages. Macrophage cell line J774A.1 (American Type Culture Collection) was maintained at $37^{\circ} \mathrm{C}$ in RPMI1640 medium (Sigma) containing 10\% heat inactivated fetal bovine serum. Briefly, J774A.1 murine macrophages $\left(1 \times 10^{5}\right.$ cells/ petridish) were infected with $1 \times 10^{6}$ promastigotes (expressing the luciferase gene (pGL$\alpha$ NEO $\alpha$ LUC) in M199 media with 10\% FBS. 
After $3 \mathrm{~h}$, the non-internalized parasites were washed off and SAG was added at different concentrations $(10-100 \mu \mathrm{g} / \mathrm{ml})$. After 5 days of drug exposure, plates containing adherent macrophages were washed and luciferase activity was determined [22]. The 50\% inhibitory concentration $\left(\mathrm{IC}_{50}\right)$ was determined from the graph representing different concentrations of SAG plotted against relative light units (RLU) produced by luciferase expressing parasites.

\section{Thiol analysis}

Thiols were derivatized with monobromobimane and separated by highperfomance liquid chromatography (HPLC) as reported earlier [18].

\section{Statistical analysis}

Data was analyzed by the Student's t- test. The data is represented as mean \pm S.D. The results are representative of three independent experiments. A $p$ value of $<0.05$ was considered statistically significant.

\section{Results}

Intracellular thiol levels in SAG-S and SAG-R clinical isolates

Resistance to antimonials in clinical isolates is not well defined. Understanding the mechanism of resistance to antimony in clinical isolates of $L$. donovani will aid in the development of biomarkers for antimony resistance and this in turn will enable the clinicians to monitor the treatment of the patients. With this background in mind, we used the previously characterized SAG-S and SAG-R, VL and PKDL clinical isolates for the present study [20].

A total of fourteen field isolates were used to assess the putative role of the $\mathrm{ABC}$ transporter MRPA, ornithine decarboxylase and $\mathrm{\gamma}$ glutamylcysteine synthetase in antimony susceptible and -resistant clinical isolates of $\mathrm{L}$. donovani from India. As reported earlier, SAG-S isolates, AG83-S, 2001-S, MC7-S, RK1-S and MS2-S coming from SAG responsive patients had $\mathrm{IC}_{50}$ values $6.2 \pm 1.8,0.9 \pm 0.12,8 \pm 3.3,0.01 \pm$ 0.02 and $4.75 \pm 0.12 \mu \mathrm{M}$ respectively whereas the SAG-R isolates, MC4-R, MC8-R, MC9-R, NR3A-
R, RMP8-R, RMP19-R, RMP142-R, RMP155-R, RMP240-R coming from the SAG-unresponsive patients had $\mathrm{IC}_{50}$ s that were $\sim 2$ to $>10$ fold higher than that of the sensitive isolate, AG83-S.(20) RK1-S was the most sensitive of all the isolates with an $\mathrm{IC}_{50}$ of $0.01 \pm 0.02 \mu \mathrm{M}$.

Intracellular thiol levels were quantified in the SAG-S and SAG-R isolates (Figure 1). SAG-R isolates maintained significantly higher levels of cysteine and glutathione as compared to the

SAG-S isolates. Cysteine levels in the SAG resistant strains MC4-R, MC8-R, MC9-R, NR3AR, RMP8-R, RMP19-R, RMP142-R, RMP155-R and RMP240-R were $\sim 1.7, \sim 1.6, \sim 1.5, \sim 2.7, \sim 2.0$, $\sim 1.8, \sim 1.8, \sim 2.0$ and $\sim 1.7$-fold higher respectively when compared to the SAG-S isolate, 2001-S (Figure 1A).

Similarly GSH levels showed significant increase in the SAG-R isolates, MC4-R ( 2.7-fold), MC8-R ( 3.6 -fold), MC9-R ( 3.0 -fold), NR3A-R ( 2.0 fold), RMP8-R ( 3.2 -fold), RMP19-R ( 2.5 -fold), RMP142-R ( 3.1 -fold), RMP155-R ( 2.4 -fold) and RMP240-R ( 3.0 -fold) when compared to the SAG sensitive isolate, 2001-S (Figure 1B). A SAG-S strain, RK1-S had glutathione levels that were 2.1 -fold higher when compared to the SAG sensitive isolate, 2001-S. Interestingly, no significant difference was observed in the trypanothione levels between the SAG-S and SAG-R isolates (Figure 1C). Similar observation was made in our earlier study using a small set of clinical isolates [23].

Gene copy number and expression profiling of the ABC transporter MRPA in SAG-S and SAG-R clinical isolates

Our earlier work on the clinical kala azar $L$. donovani isolates from India showed MRPA overexpression as an important SAG resistance factor [23]. To further validate, the role of $M R P A$ gene in antimony resistance phenotype, we checked the amplification of MRPA gene by Southern blot hybridization. Southern blot hybridization of total genomic DNA digested with HindIII followed by hybridization with 

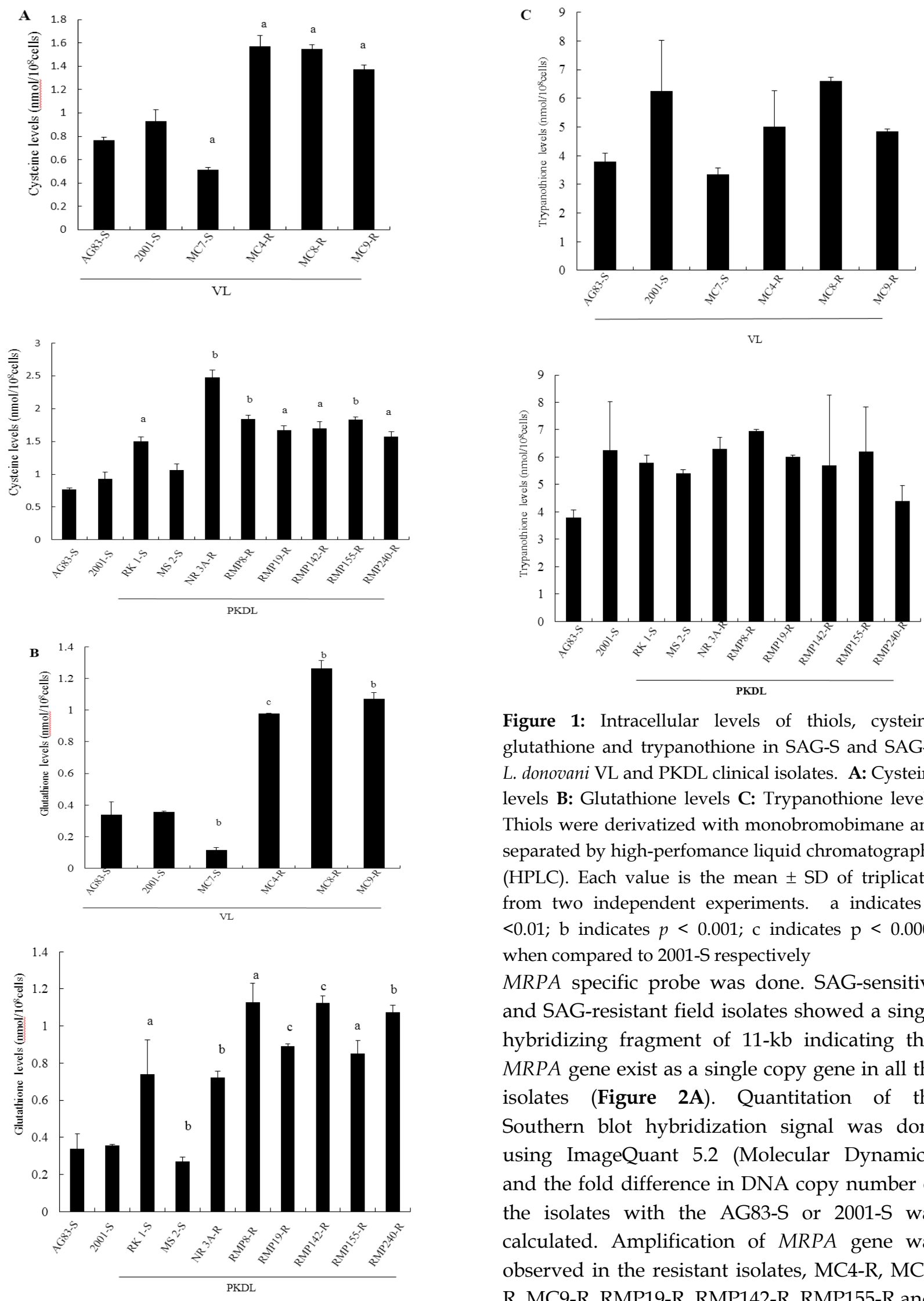

Figure 1: Intracellular levels of thiols, cysteine, glutathione and trypanothione in SAG-S and SAG-R L. donovani $\mathrm{VL}$ and PKDL clinical isolates. A: Cysteine levels B: Glutathione levels C: Trypanothione levels. Thiols were derivatized with monobromobimane and separated by high-perfomance liquid chromatography (HPLC). Each value is the mean \pm SD of triplicates from two independent experiments. a indicates $p$ $<0.01$; $\mathrm{b}$ indicates $p<0.001$; $\mathrm{c}$ indicates $\mathrm{p}<0.0004$ when compared to 2001-S respectively

MRPA specific probe was done. SAG-sensitive and SAG-resistant field isolates showed a single hybridizing fragment of $11-\mathrm{kb}$ indicating that MRPA gene exist as a single copy gene in all the isolates (Figure 2A). Quantitation of the Southern blot hybridization signal was done using ImageQuant 5.2 (Molecular Dynamics) and the fold difference in DNA copy number of the isolates with the AG83-S or 2001-S was calculated. Amplification of MRPA gene was observed in the resistant isolates, MC4-R, MC8R, MC9-R, RMP19-R, RMP142-R, RMP155-R and 
$\mathbf{A}$

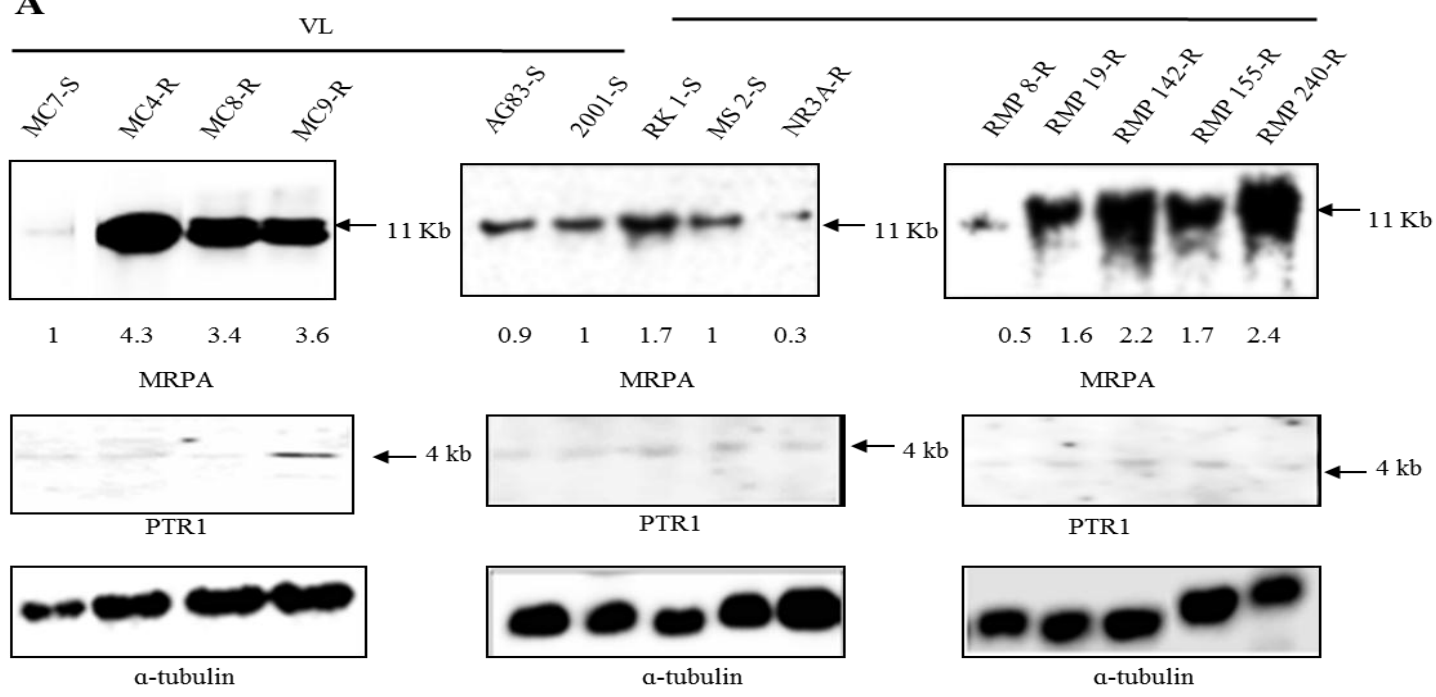

\section{B}

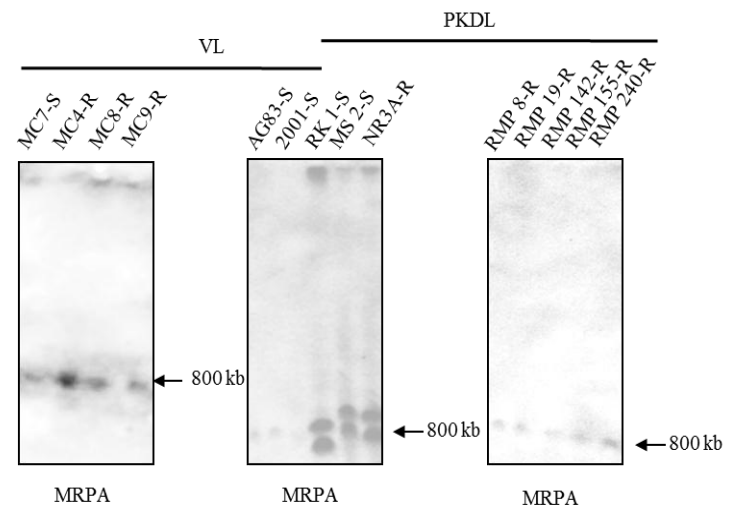

C

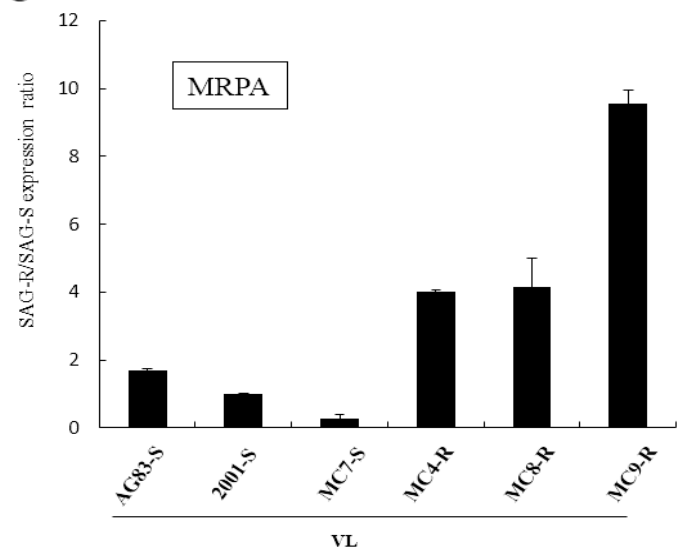

Figure 2: A: Southern blot analysis of MRPA and PTR1 genes in SAG-S and SAG-R L. donovani VL and PKDL clinical isolates. Total genomic DNA of isolates were digested with HindIII, electrophoresed, blotted and hybridized with a MRPA specific probe of 400-bp and $6 \mathrm{~kb}$ 5'-PTR1 specific probe. The size of the hybridizing bands was determined using HindIII

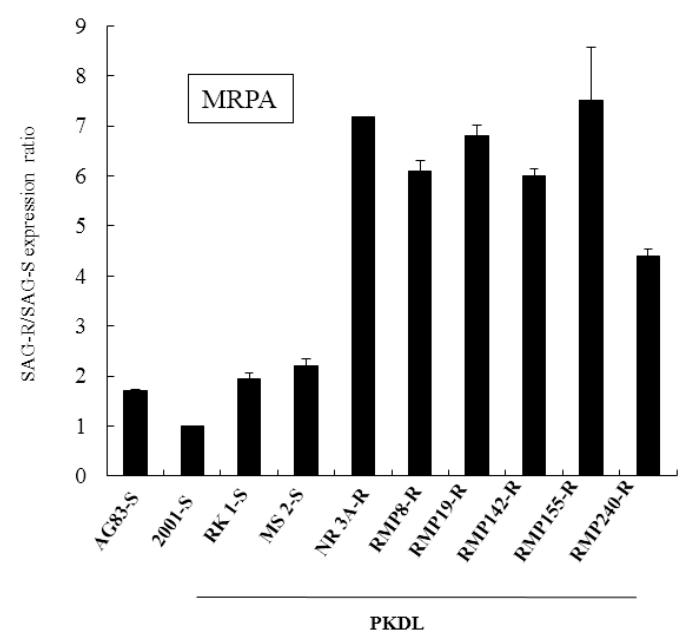

digested lambda DNA. The blot was rehybridized with a-tubulin probe to monitor the amount of digested DNA layered on the gel. Quantitation of Southern blot was done by Image-quant software 5.2 (Molecular dynamics) and the fold difference in DNA copy number of MRPA is presented below each blot. B: Southern blot analysis of the pulse field gel electrophoresis of SAG-sensitive (SAG-S) and SAGresistant (SAG-R) isolates of Leishmania donovani chromosomes. Agarose blocks containing chromosomal DNAs of promatigotes of L. donovani clinical isolates were prepared and subjected to pulsed field gel electrophoresis for 24 hours run time and hybridized with a MRPA specific probe of 400-bp. C: Real time RT-PCR expression analysis of MRPA gene in L. donovani clinical isolates. MRPA RNA expression ratios in promastigotes of SAG -resistant isolates are relative to the SAG-sensitive isolate (2001-S). The graph represents mean of three independent experiments performed from three different RNA preparations. 
PKDI
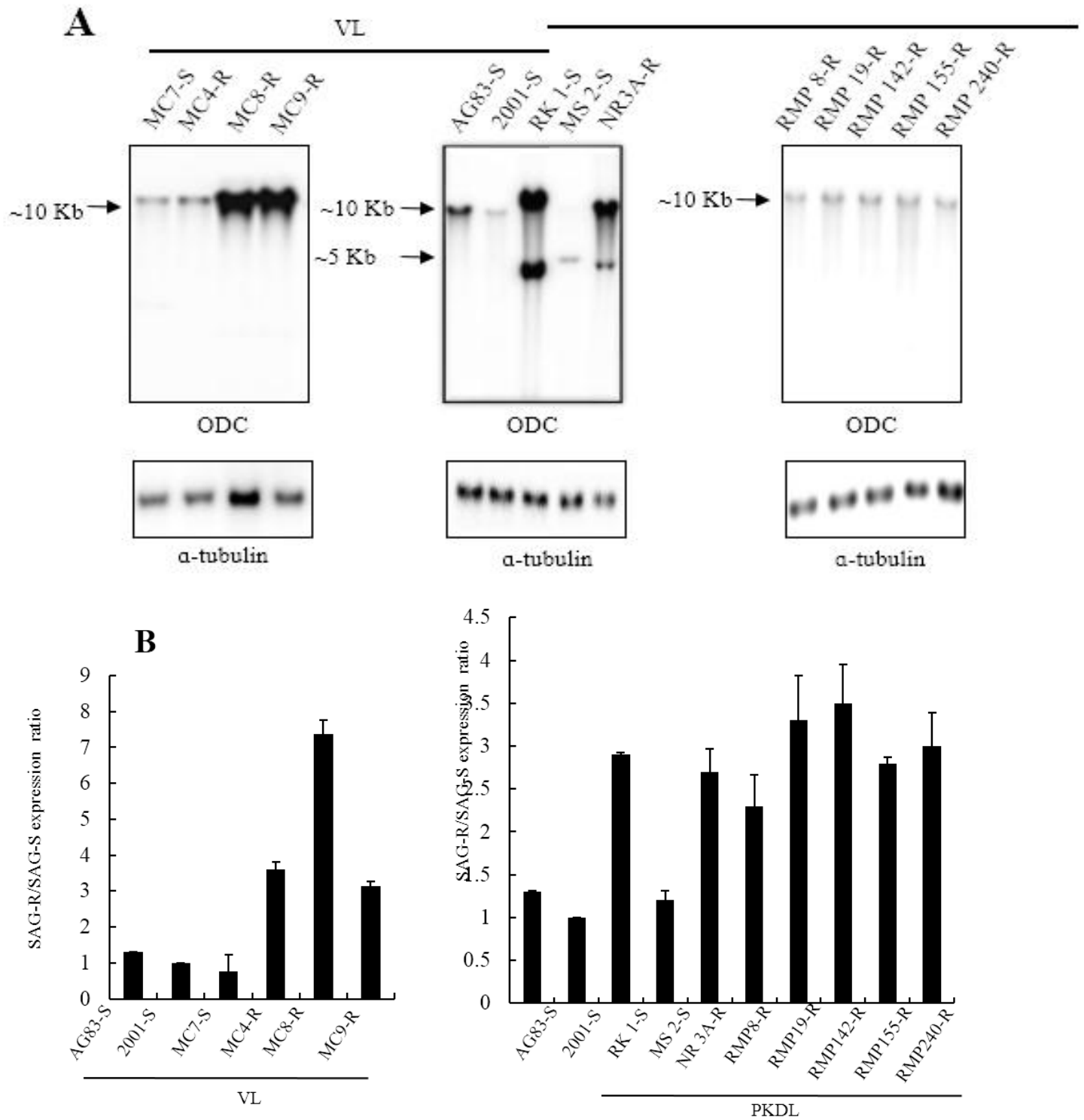

Figure 3: A: Characterization of ODC gene in SAG-S and SAG-R isolates L. donovani VL and PKDL clinical isolates. Genomic DNAs were isolated and digested with HindIII and hybridized with a full-length ODC specific probe, derived from the L. donovani ODC gene. The sizes of the hybridizing bands were determined using HindIII digested lambda DNA marker. The blot was rehybridized with a-tubulin probe to monitor the amount of digested DNA layered on the gel. B: Real time RT-PCR expression analysis of ODC in L. donovani clinical isolates. ODC RNA expression ratios in the SAG- resistant isolates are relative to the SAG-sensitive isolate, 2001-S. Results are mean of three independent experiments performed from three different RNA preparations.

RMP240-R (Figure 2A). No amplification was observed in the resistant isolates, NR3A-R and RMP8-R (Figure 2A). The amplification observed in the resistant isolates was further analyzed by PFGE (Figure 2B). None of the resistant isolates showed circular amplification as was observed in our earlier studies with limited number of isolates [23]. Interestingly, two sensitive PKDL isolates, RK1-S and MS2-S and one resistant
PKDL isolate, NR3A-R had the presence of MRPA on two chromosomes as indicated by their characteristic migration in PFGE (Figure 2B). We had earlier reported co-amplification of pterin reductase gene (PTR1) with MRPA in the clinical isolates [23]. In the present study, coamplification of PTR1 gene with MRPA gene was not observed in any of the clinical isolates as 

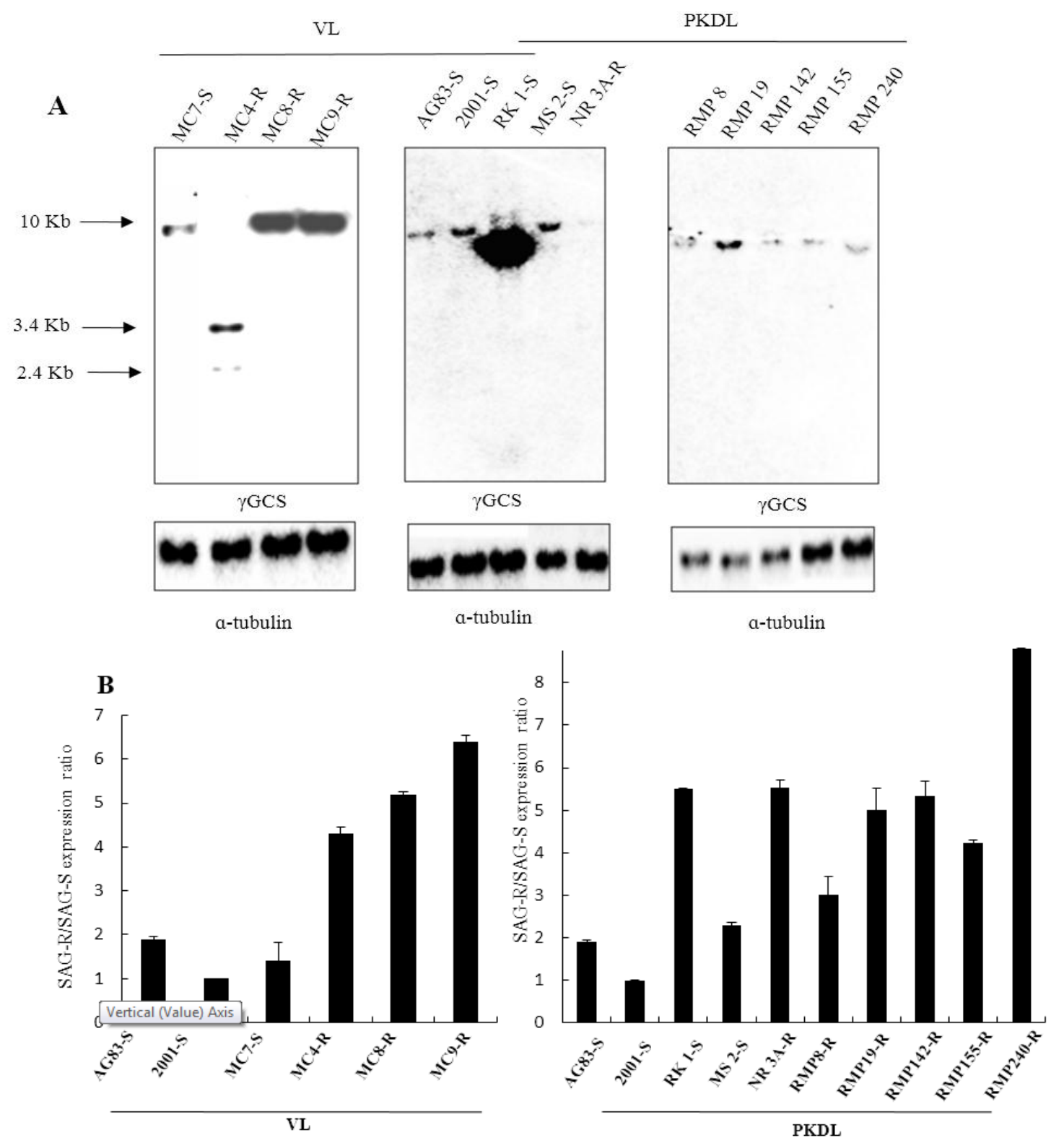

Figure 4: A: Southern blot analysis of the $\gamma$-GCS gene in the SAG-sensitive and the SAG-resistant Leishmania donovani VL and PKDL field isolates. Total genomic DNA was isolated and digested with HindIII. The digested DNA was electrophoresed, blotted and hybridized with $\gamma$-GCS probe. The sizes of the hybridizing bands were determined using HindIII digested $\lambda$ - DNA. The blot was rehybridized with a-tubulin probe to monitor the amount of digested DNA layered on the gel. B: Real time RT-PCR expression analysis of $\gamma$-GCS gene in L. donovani clinical isolates. $\gamma$-GCS gene expression ratios in the SAG-resistant isolates are relative to the SAG- sensitive isolate, 2001-S. Results are mean of three independent experiments performed from three different RNA preparations.

determined by Southern-blot analysis using PTR1-specific probe (Figure 2A).

Comparison of MRPA gene expression in SAG-S versus SAG-R field isolates was done to verify if there is any correlation between MRPA gene expression and SAG susceptibility profile of the clinical isolates. Total RNA from promastigotes of the clinical isolates was isolated and complementary DNAs were synthesized. Realtime -PCR using QuantiFast SYBR Green PCR Master Mix (Qiagen) with MRPA (gene specific) and GAPDH (internal control) primers was performed. Up-regulation of MRPA expression was observed in the resistant clinical isolates. $M R P A$ expression in the resistant strains, MC4R, MC8-R, MC9-R, NR3A-R, RMP8-R, RMP19-R, 
RMP142-R, RMP155-R and RMP240-R was $\sim 4-$, $\sim 4.2_{-}^{-}, \sim 9.6-, \sim 7.2-, \sim 6.1-, \sim 6.8-, \sim 6.0-$, $\sim 7.5-$ and $\sim 4$.4- fold more respectively when compared to the expression in the sensitive isolate, 2001-S (Figure 2C).

Gene copy number and expression profiling of the ornithine decarboxylase (ODC) gene in SAG-S and SAG-R clinical isolates.

Ornithine decarboxylase (ODC) is the ratelimiting enzyme of the polyamine biosynthetic pathway. In addition to MRPA, overexpression of the ODC gene has been reported in the antimony resistant mutants [23-25]. These observations prompted us to determine if amplification of the ODC gene occured in our clinical isolates. Southern-blot analysis of the total genomic DNA digested with HindIII and hybridized with the ODC specific probe was done. SAG-S and SAG-R isolates showed a single copy of the ODC gene (Figure 3A) with the exception of three PKDL isolates, RK1-S, MS2-S and NR3A-R. Interestingly, two copies of the $O D C$ gene were observed in these three PKDL isolates (Figure 3A).

Comparison of ODC gene expression in SAG-S versus SAG-R field isolates was done to verify if there is any correlation between the gene expression and SAG susceptibility profile of the clinical isolates. Real-time PCR with the ODC (gene specific) and the GAPDH (internal control) primers was performed. Up-regulation of the $O D C$ expression was observed in the resistant clinical isolates. ODC expression in the resistant strains, MC4-R, MC8-R, MC9-R, NR3A-R, RMP8R, RMP19-R, RMP142-R, RMP155-R and RMP240-R was 3.6-, 7.4-, 3.1-, 2.7-, 2.3-, $\sim 3.3-, \sim 3.5-, \sim 2.8-$ and $\sim 3.0-$ fold more respectively when compared to the sensitive isolate, 2001-S (Figure 3B). SAG-S isolate, RK1-S was an exception since it was the only sensitive isolate that showed 2.9-fold up-regulation when compared to the reference strain, 2001-S and also in comparison with all other SAG-S isolates (Figure 3B). No uniform correlation was observed between gene amplification and $O D C$ gene expression in these isolates.
Gene copy number and expression profiling of the $\gamma$-glutamylcysteine synthetase $(\gamma$-GCS $)$ gene in SAG-S and SAG-R clinical isolates

In addition to MRPA and ODC, another locus that has been reported to be amplified in the antimony-resistant isolates is the GSH1 gene coding for the heavy subunit of $\gamma$-GCS. $\gamma$-GCS is the rate limiting enzyme for GSH synthesis $[14,23]$. We performed Southern blot analysis of the total genomic DNA digested with HindIII and hybridized with the $\gamma$-GCS specific probe. Southern blot analysis of the $\gamma$-GCS gene showed two copies in a resistant isolate MC4-R but in all other isolates, $\gamma$-GCS probe hybridized to a single hybridizing fragment of $10-\mathrm{kb}$ (Figure 4A). SAG-S isolate, RK1-S was again an exception since it showed amplification of the $\gamma$-GCS gene (Figure 4A).

Comparison of $\gamma$ GCS gene expression in SAG-S versus SAG-R field isolates was done to verify if there is any correlation between gene expression and SAG sensitivity profile of the clinical isolates. Real-time PCR using $\gamma$-GCS (gene specific) and GAPDH (internal control) primers was performed. Up-regulation of $\gamma$-GCS expression was observed in the resistant clinical isolates. $\gamma$-GCS expression in the resistant strains, MC4-R, MC8-R, MC9-R, NR3A-R, RMP8-R, RMP19-R, RMP142-R, RMP155-R and RMP240-R was 4.3-, 5.2-, 6.4-, 5.5-, 3.0-, 5.0-, 5.3-, 4.2- and 8.8- fold more respectively compared to the expression in the sensitive isolate, 2001-S (Figure 4B). In the present study though upregulation of $\gamma$-GCS was observed in all SAG-R isolates, interestingly one SAG-S isolate, RK1-S showed 5.5-fold up-regulation of $\gamma$ GCS expression when compared to a SAG-S isolate, 2001-S (Figure 4B). L-butathione-sulfoxamine (BSO), an inhibitor of $\gamma$-GCS was used to compare its effect on the SAG-S isolate, RK1-S and 2001-S. RK1-S promastigotes overexpressing $\gamma$-GCS were $\sim 5$ fold more resistant to BSO when compared to the $\mathrm{IC}_{50}$ of promastigotes of the 2001-S, the $\mathrm{IC}_{50}$ being $6.5 \pm$ $0.5 \mathrm{mM}$ and $1.3 \pm 0.3 \mathrm{mM}$ respectively. 

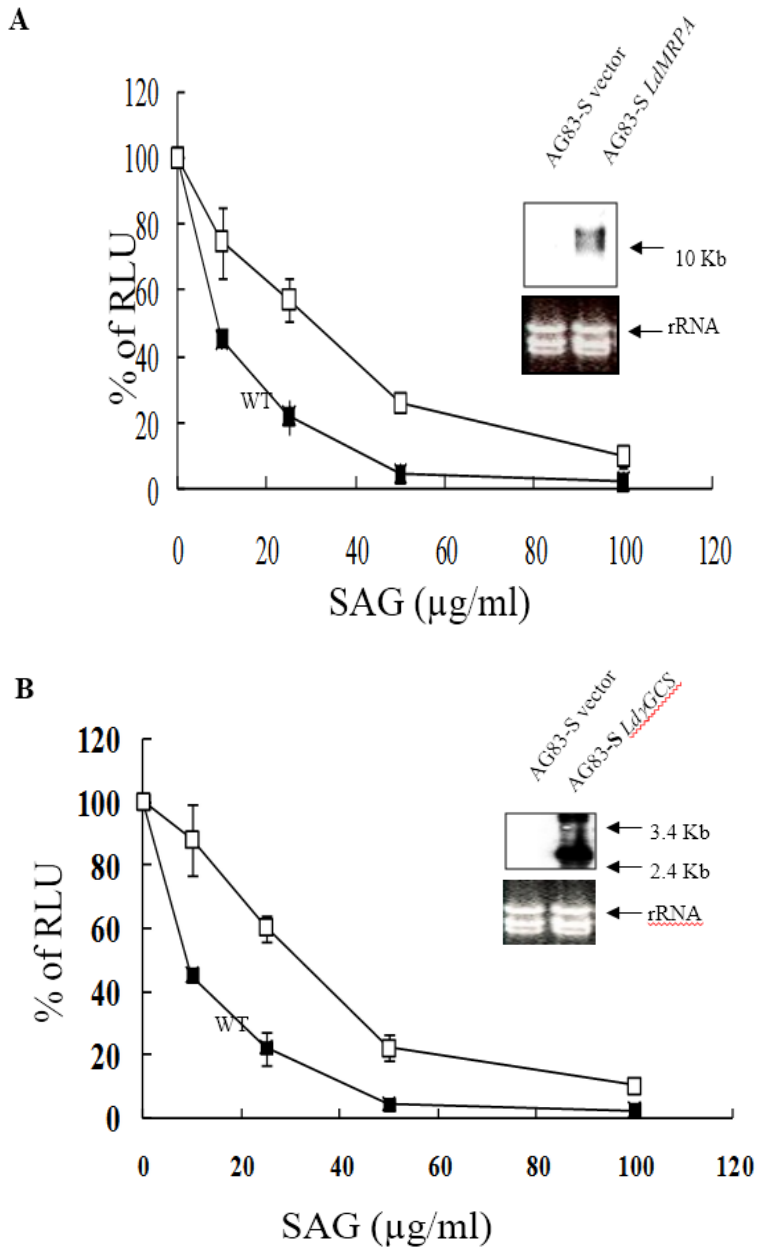

Figure 5: Over-expression of MRPA and $y$-GCS gene in a sensitive $L$. donovani isolate. A: Transfection of MRPA in SAG-sensitive isolate leads to resistance to antimony in amastigotes. AG83-S (- - -) was transfected with pGL- $\alpha$ NEO $\alpha$ LUC, AG83-S + LdMRPA (- -$)$ was transfected with pGEM72f- $\alpha$ neo $\alpha-M R P A+$ pGL$\alpha$ NEO $\alpha$ LUC and sensitivity to SAG in the J774A.1 line was measured as described in Materials and methods. Each data point represents the mean \pm SD of three determinations. Northern blot analysis (insets in A) of vector transfected controls and MRPA transfected lines was performed as described under methods section B: Transfection of $\mathrm{y}$-GCS gene in SAGsensitive isolate leads to antimony resistance in intracellular amastigotes. AG83-S (-m-) transfected with pGL- $\alpha$ NEO $\alpha$ LUC, AG83-S + y-GCS (-口-) transfected with pspahygro $\alpha-\gamma \mathrm{GCS}+$ pGL$\alpha$ NEO $\alpha$ LUC and sensitivity to SAG measured as described in Materials and methods. Each data point represents the mean $\pm \mathrm{SD}$ of three determinations. Northern blot analysis (insets in B) of vector transfected controls and $\gamma$-GCS transfected lines was performed as described under methods section.
Overexpression of MRPA or $\gamma$-GCS in an antimony-sensitive isolate conferred increased expression and resistance to antimony

To determine whether overexpression of MRPA and $\gamma$-GCS conferred antimony resistance in a sensitive isolate, we transfected MRPA or $\gamma$-GCS constructs into a SAG-S isolate, AG83-S. These MRPA and $\gamma$-GCS recombinant parasites were co-transfected with pGL- $\alpha$ NEO $\alpha \mathrm{LUC}$, encoding LUC. Intracellular amastigotes over-expressing MRPA $\left(\mathrm{IC}_{50}, 32.3 \pm 6.4 \mu \mathrm{g} / \mathrm{ml}\right)$ were 3.6 -fold more resistant to SAG when compared to amastigotes of the parent strain transfected with the control vector $\left(\mathrm{IC}_{50}, 9 \pm 0.5 \mu \mathrm{g} / \mathrm{ml}\right.$ ) (Figure 5A). Intracellular amastigotes over-expressing $\gamma$ GCS were 3.3-fold resistant to SAG when compared to the sensitive $L$. donovani the $\mathrm{IC}_{50}$ value being $(29.7 \pm 1.5 \mu \mathrm{g} / \mathrm{ml})$ respectively (Figure 5B). In our previous studies we had demonstrated that the ODC overexpressors exhibited significant resistance to Pentostam compared to the wild type cells (26). Intracellular amastigotes over-expressing $\mathrm{ODC}\left(\mathrm{IC}_{50},>80\right.$ $\mu \mathrm{g} / \mathrm{ml}$ ) were $>8.8$-fold more resistant to SAG when compared to amastigotes of the parent strain transfected with the control vector [26].

\section{Discussion}

Currently, chemotherapy is the only effective way to control Leishmania infection. Pentavalent antimonials are the mainstay of therapy in the treatment of visceral leishmaniasis [27]. Increase in resistance to SAG has led to an upsurge in therapeutic failure and in the absence of limited chemotherapeutic alternatives, it is extremely necessary to identify biomarkers for monitoring antimony resistance.

Trivalent form of the antimonial drug (SbIII) is the prodrug that is formed by conversion of pentavalent antimony $(\mathrm{SbV})$ by a putative metalloid reductase present in the macrophages [28]. Antimonial resistance in both laboratory mutants and clinical isolates has been associated with (a) decreased uptake of the drug through aquaglyceroporin (AQP1) that codes for the protein responsible for SbIII transport 
$[11,12,20,29]$ (b) Over expression of ODC and $\gamma^{-}$ GCS enzymes of the trypanothione biosynthetic pathway [2,25] and (c) increased expression of the $A B C$ transporter MRPA, which sequesters SbIII-thiol conjugate $[16,23]$.

We had earlier reported decreased uptake of antimony in all nine SAG-R isolates used in the present study. Down-regulation of SbIII influx pump; aquaglyceroporin (AQP1) was observed in seven out of the nine resistant isolates Strains, MC8-R and NR3A-R were an exception since they showed up-regulation of $A Q P 1$ gene expression [20].

The ABC transporter gene MRPA causes drug sequestration in Leishmania promastigotes and amastigotes selected for SbIII resistance [17,30]. Increased copy number of MRPA has been reported in SbIII resistant mutant [2]. Our earlier studies using limited number of SAG-S and SAG-R clinical isolates showed amplification of MRPA gene as part of an extrachromosomal circle [23]. In the present study, we found distinct correlation between copy number of $M R P A$ gene and antimony sensitivity in a majority of the isolates (Figure 2A). However, two SAG-R strains, NR3A-R and RMP8-R were an exception. We have reported earlier that $A B C$ transporter gene MRPA was amplified in three out of four resistant VL isolates as part of the extrachromosmal circle and coamplifcation of PTR1 along with MRPA suggested amplification of the $\mathrm{H}$ locus in SAG-resistant clinical isolates [23]. In the present study, co-amplification of PTR1 gene with MRPA gene was not observed in any of the clinical isolates. CHEF gel analysis of the SAG-S and SAG-R isolates did not show any circular amplification in any of the SAG-R isolates. MRPA gene expression in SAG-S versus SAG-R field isolates showed correlation between $M R P A$ gene expression and SAG susceptibility profile of the clinical isolates. The present observation validated our earlier results where we have shown correlation between MRPA gene expressions with the antimony resistant clinical profile in the field conditions [23].

(ONJB, Biotechnology Society of Nepal
In our previous study amplification of $O D C$ gene was noted in the resistant isolates but not that of the $\gamma$-GCS [23]. In another study on L. donovani isolates from Nepal, expression of $\gamma$-GCS and $O D C$ was significantly decreased in the resistant isolates [31]. In the present study we observed increased expression of $O D C$ gene and $\gamma$-GCS in all SAG-R isolates.

It has been reported earlier that $\mathrm{ABC}$ transporter MRPA, confers resistance to antimonials by sequestration of metal thiol conjugates in an intracellular organelle located close to the flagellar pocket [16]. This model has been demonstrated in promastigotes of L. tarentolae, in amastigotes and also in clinical isolates from India $[16,23,30]$. These observations clearly highlighted the importance of intracellular thiol in MRPA mediated efflux of the antimony. An increase in cysteine and glutathione levels were reported in antimony resistant $L$. donovani clinical isolates [23]. In the present study also, we observed increase in cysteine and glutathione levels in all SAG-R isolates. However, no change in trypanothione levels were observed in SAG-R isolates in comparison to SAG-S isolates. Similar observation was made in our analysis of the mode of action of antimony in clinical isolates and earlier studies in L. infantum resistant to $\mathrm{Sb}$ (III) [23]. It was pointed out that antimony possibly depleted trypanothione by efflux of Sbtrypanothione conjugate [16]. It is possible that the efflux system is increased in the resistant isolates thereby leading to increased trypanothione efflux. This would explain the constant levels of trypanothione in the present study in the resistant isolates.

RK1-S though a SAG-S isolate, was an exception to the mutlifactorial antimony resistant mechanisms reported in clinical isolates and also in the lab based resistant isolates. Clinical history of the RK1-S patient showed that the interval between the cure of VL and the onset of PKDL was 2.5 years where as MS2-S and NR3A-R, PKDL isolates the interval between the cure of VL and the onset of PKDL was 7 and 11 years respectively. It will be interesting to look at the 
PKDL isolates with known clinical history in order to determine if this interval has a role to play in antimony susceptibility/resistance.

The data presented here in VL and for the first time in PKDL isolates, establishes the relevance of overexpression of $\gamma$-GCS and ODC and an increased expression of MRPA that may be responsible for an increased efflux of thiol-Sb-III conjugate. Our data further confirms that resistance mechanisms present in the laboratory strains can be found in the clinical isolates.

Further work is presently going on the laboratory to get a global overview of the resistant mechanism by proteomics approach in order to find other possible resistant determinants. It will further help in proper selection of therapeutic regimen and improve treatment strategy against leishmaniasis.

\section{Acknowledgements}

The clinical isolates used in this study were kindly provided by Dr. Mitali Chatterjee (Institute of Post Graduate Medical Education and Research, Kolkata, India) and Dr. Sarman Singh, (Division of Clinical Microbiology, All India Institute of Medical Sciences, New Delhi, India).

\section{Funding}

This work is supported by a grant from the Department of Biotechnology to Dr. Rentala Madhubala. Mahendra Maharjan and Swati Mandal are supported by a fellowship from the University Grants Commission, Government of India

Transparency declarations: None to declare.

\section{References}

1. Guerin PJ, Olliaro P, Sundar S, Boelaert M, Croft SL, Desjeux P, Wasunna MK, Bryceson AD: Visceral leishmaniasis: current status of control, diagnosis, and treatment, and a proposed research and development agenda. Lancet Infect Dis. 2002, 2(8):494501.

2. Haimeur A, Brochu C, Genest P, Papadopoulou B, Ouellette M: Amplification of the ABC transporter gene PGPA and increased trypanothione levels in potassium antimonyl tartrate (SbIII) resistant Leishmania tarentolae. Mol Biochem Parasitol. 2000, 108(1):131-135.

CNJB, Biotechnology Society of Nepal
3. Sundar S, More DK, Singh MK, Singh VP, Sharma S, Makharia A, Kumar PC, Murray HW: Failure of pentavalent antimony in visceral leishmaniasis in India: report from the center of the Indian epidemic. Clin. Infect. Dis. 200, 31(4): 1104-1107.

4. Thakur CP, Kumar K: Post kala-azar dermal leishmaniasis: a neglected aspect of kala-azar control programmes. Ann Trop Med Parasitol. 1992, 86(4): 355-359.

5. Zijlstra EE, Musa AM, Khalil EA, el-Hassan IM, elHassan AM: Post-kala-azar dermal leishmaniasis. Lancet Infect. Dis. 2003, 3(2): 87-98.

6. Roberts $\mathrm{WL}$, Berman JD, Rainey $\mathrm{PM:}$ In vitro antileishmanial properties of tri- and pentavalent antimonial preparations. Antimicrob Agents Chemother. 1995, 39(6): 1234-1239.

7. Denton H, McGregor JC, Coombs GH: Reduction of anti-leishmanial pentavalent antimonial drugs by a parasite-specific thiol-dependent reductase, TDR1. Biochem J. 2004, 381(2): 405-412.

8. Ferreira CS, Martins PS, Demicheli C, Brochu C, Ouellette $M$, Frezard F: Thiol-induced reduction of antimony(V) into antimony(III): a comparative study with trypanothione, cysteinyl-glycine, cysteine and glutathione. Biometals 2003, 16(3): 441446.

9. Zhou Y, Messier N, Ouellette M, Rosen BP, Mukhopadhyay R: Leishmania major LmACR2 is a pentavalent antimony reductase that confers sensitivity to the drug pentostam. J Biol Chem. 2004, 279: 37445-37451.

10. Shaked-Mishan P, Ulrich N, Ephros M, Zilberstein D: Novel Intracellular $\mathrm{SbV}$ reducing activity correlates with antimony susceptibility in Leishmania donovani. J Biol Chem. 2001, 276(6): 3971-3976.

11. Gourbal B, Sonuc N, Bhattacharjee H, Legare D, Sundar S, Ouellette M, Rosen BP, Mukhopadhyay R: Drug uptake and modulation of drug resistance in Leishmania by an aquaglyceroporin. J Biol Chem. 2004, 279(30): 31010-31017.

12. Marquis N, Gourbal B, Rosen BP, Mukhopadhyay R, Ouellette M: Modulation in aquaglyceroporin $A Q P 1$ gene transcript levels in drug-resistant Leishmania. Mol Microbiol. 2005, 57(6): 1690-1699.

13. Wyllie S, Cunningham ML, Fairlamb AH: Dual action of antimonial drugs on thiol redox metabolism in the human pathogen Leishmania donovani. J Biol Chem. 2004, 279(38): 39925-39932.

14. Dey S, Ouellette M, Lightbody J, Papadopoulou B, Rosen BP: An ATP-dependent As (III)-glutathione transport system in membrane vesicles of Leishmania tarentolae. Proc Natl Acad Sci U S A. 1996, 93(5), 2192-2197.

15. Fairlamb AH, Cerami A: Metabolism and functions of trypanothione in the Kinetoplastida. Annu Rev Microbiol. 1992, 46: 695-729.

16. Legare D, Richard D, Mukhopadhyay R, Stierhof YD, Rosen BP, Haimeur A, Papadopoulou B, Ouellette M: 
The Leishmania ATP-binding cassette protein PGPA is an intracellular metal-thiol transporter ATPase. J Biol Chem. 2001, 276(28): 26301-26307.

17. Legare D, Papadopoulou B, Roy G, Mukhopadhyay R, Haimeur A, Dey S, Grondin K, Brochu C, Rosen $B P$, Ouellette M: Efflux systems and increased trypanothione levels in arsenite-resistant Leishmania. Exp Parasitol. 1997, 87(3): 275-282.

18. Mukhopadhyay R, Dey S, Xu N, Gage D, Lightbody J, Ouellette M, Rosen BP: Trypanothione overproduction and resistance to antimonials and arsenicals in Leishmania. Proc. Natl. Acad. Sci. U. S. A 1996, 93(19): 10383-10387.

19. Dey A, Singh S: Genetic heterogeneity among visceral and post-Kala-Azar dermal leishmaniasis strains from eastern India. Infect Genet Evol. 2007, 7(2): 219-222.

20. Mandal S, Maharjan $M$, Singh $S$, Chatterjee $M$, Madhubala R: (2010) Assessing aquaglyceroporin gene status and expression profile in antimonysusceptible and -resistant clinical isolates of Leishmania donovani from India. J Antimicrob Chemother. 2010, 65(3): 496-507.

21. Sambrook J, Fritsch EF, Maniatis T: Molecular cloning: A laboratory manual. Cold Spring Harbour Press. Int J Hyperthermia. 1989, 8: 329-340

22. Roy G, Dumas C, Sereno D, Wu Y, Singh AK, Tremblay MJ, Ouellette M, Olivier M, Papadopoulou B: Episomal and stable expression of the luciferase reporter gene for quantifying Leishmania spp. infections in macrophages and in animal models. Mol Biochem Parasitol. 2000, 110(2): 195-206.

23. Mukherjee A, Padmanabhan PK, Singh S, Roy G, Girard I, Chatterjee M, Ouellette, M, Madhubala R: Role of $\mathrm{ABC}$ transporter MRPA, gammaglutamylcysteine synthetase and ornithine decarboxylase in natural antimony-resistant isolates of Leishmania donovani. J Antimicrob Chemother. 2007, 59(2): 204-211.

24. Grondin K, Haimeur A, Mukhopadhyay R, Rosen BP, Ouellette $\mathrm{M}$ : Co-amplification of the gammaglutamylcysteine synthetase gene gsh1 and of the $\mathrm{ABC}$ transporter gene pgpA in arsenite-resistant Leishmania tarentolae. EMBO J. 1997, 16(11): $3057-$ 3065.

25. Haimeur A, Guimond C, Pilote S, Mukhopadhyay R, Rosen BP, Poulin R, Ouellette M: Elevated levels of polyamines and trypanothione resulting from overexpression of the ornithine decarboxylase gene in arsenite-resistant Leishmania. Mol Microbiol. 1999, 34(4): 726-735.

26. Singh S, Mukherjee A, Khomutov AR, Persson L, Heby O, Chatterjee M, Madhubala R: Antileishmanial effect of 3-aminooxy-1- aminopropane is due to polyamine depletion. Antimicrob Agents Chemother. 2007, 51(2): 528-534.

27. den Boer ML, Alvar J, Davidson RN, Ritmeijer K, Balasegaram M: Developments in the treatment of visceral leishmaniasis. Expert Opin Emer. Drugs. 2009, 14(3): 395-410.

28. Sereno D, Cavaleyra M, Zemzoumi K, Maquaire S, Ouaissi A, Lemesre JL: Axenically grown amastigotes of Leishmania infantum used as an in vitro model to investigate the pentavalent antimony mode of action. Antimicrob Agents Chemother. 1998, 42(12): 3097-3102.

29. Maharjan M, Singh S, Chatterjee M, Madhubala R: Role of aquaglyceroporin (AQP1) gene and drug uptake in antimony-resistant clinical isolates of Leishmania donovani. Am J Trop Med Hyg. 2008, 79(1): 69-75.

30. El FK, Messier N, Leprohon P, Roy G, Guimond C, Trudel N, Saravia NG, Papadopoulou B, Legare D, Ouellette M: Role of the ABC transporter MRPA (PGPA) in antimony resistance in Leishmania infantum axenic and intracellular amastigotes. Antimicrob Agents Chemother. 2005, 49(5): 1988-1993.

31. Decuypere S, Rijal S, Yardley V, De DS, Laurent T, Khanal B, Chappuis F, Dujardin JC: Gene expression analysis of the mechanism of natural $\mathrm{Sb}(\mathrm{V})$ resistance in Leishmania donovani isolates from Nepal. Antimicrob Agents Chemother. 2005, 49(11): 4616-4621. 